\title{
Unrecognized obstructive sleep apnea in surgery: we can't afford to sleep on it any longer
}

\author{
Aaron $\mathrm{Oh}^{1}$, Sutapa Mukherjee ${ }^{1,2}$, R. Doug McEvoy ${ }^{1,2}$ \\ ${ }^{1}$ Respiratory and Sleep Service, Southern Adelaide Local Health Network, Bedford Park, SA, Australia; ${ }^{2}$ Adelaide Institute for Sleep Health, College \\ of Medicine and Public Health, Flinders University, South Australia, Australia \\ Correspondence to: R. Doug McEvoy. Respiratory and Sleep Service, Southern Adelaide Local Health Network, Bedford Park 5042 SA, Australia. \\ Email: doug.mcevoy@flinders.edu.au. \\ Provenance: This is an invited article commissioned by the Academic Editor Hao Zhang (Rocket Force Characteristic Medical Center of PLA, \\ Beijing, China). \\ Comment on: Chan MT, Wang CY, Seet E, et al. Association of Unrecognized Obstructive Sleep Apnea With Postoperative Cardiovascular Events in \\ Patients Undergoing Major Noncardiac Surgery. JAMA 2019;321:1788-98.
}

Submitted Aug 22, 2019. Accepted for publication Sep 27, 2019.

doi: $10.21037 /$ jtd.2019.10.11

View this article at: http://dx.doi.org/10.21037/jtd.2019.10.11

Obstructive sleep apnea (OSA) is characterized by cyclical upper airway collapse, hypoxia, arousal from sleep, and is associated with sympathetic discharge, endothelial dysfunction, and hypercoagulability (1). Severe, untreated OSA is associated with adverse cardiovascular (CV) events, and is under-diagnosed in the community (2). The prevalence of OSA in surgical populations is high, and current anesthetic guidelines recommend preoperative screening for OSA (3). In the postoperative period, the combined effects of anesthesia, sedation and analgesia conspire to exacerbate pre-existing OSA. What is less clearly understood is the effect of OSA on postoperative outcomes, with previous analyses of large databases showing conflicting results (4-6).

The Postoperative Vascular Complications in Unrecognized OSA (POSA) study by Chan et al. was designed to address this shortcoming (7). It investigated whether there was an association between previously undiagnosed and untreated OSA and a composite primary outcome of adverse CV events (myocardial injury, heart failure, atrial fibrillation, stroke, thromboembolism, cardiac death) in the 30-day period after surgery. The study was large $(n=1,218)$, prospective in design with an international, multi-center and multi-ethnic reach, and had rigorous follow-up. Recruited patients were $\geq 45$ years old with one or more risk factors for postoperative $\mathrm{CV}$ complications (e.g., existing peripheral, cardio- or cerebrovascular disease, treated diabetes, heart or renal failure) who were undergoing major non-cardiac surgery. Pre-operative limited-channel sleep monitoring was used to measure the apnea hypopnea index (AHI, number of apneas and hypopneas per hour of recording) and stratify participants into mild (AHI 5-14.9), moderate (AHI 15-30), and severe (AHI >30) OSA groups. The prevalence of OSA (defined as all participants with $\mathrm{AHI}>5$ ) was high $(68 \%)$, although considerably smaller proportions of the study population had severe $(11 \%)$ and moderate $(19 \%)$ OSA. The primary composite CV outcome occurred in 19\% of participants. After adjustment for known adverse postoperative CV event risk factors, a statistically significant association was found between severe OSA and the primary outcome [adjusted hazard ratio (HR) 2.23; 95\% CI, 1.49-3.34]; $\mathrm{P}=0.001$ ), but not in participants with mild (adjusted HR 1.36; $95 \%$ CI, 0.97-1.91; $\mathrm{P}=0.08$ ) or moderate OSA (adjusted HR 1.47; 95\% CI, 0.98-2.09; $\mathrm{P}=0.07$ ). Notably, participants with severe OSA had a 13-fold higher risk of cardiac death (adjusted HR 13.56; 95\% CI, 1.60-114.19; P=0.02), a 7-fold higher risk of heart failure (adjusted HR 7.04; $95 \%$ CI, 1.86-26.66; $\mathrm{P}=0.004)$, and a nearly 4-fold higher risk of new-onset atrial fibrillation (adjusted HR 3.75; 95\% CI, $1.19-11.87 ; \mathrm{P}=0.03)$.

A significant proportion of all recorded CV events $(124 / 235,53 \%)$ were ascribed to myocardial injury, defined by the authors as "elevated cardiac troponin values within 30 days after surgery without evidence of a non-ischemic etiology, such as pulmonary embolism, sepsis, cardioversion, or known 
persistent elevated cardiac troponin values". A postoperative high-sensitivity troponin elevation in patients undergoing non-cardiac surgery is associated with 30-day mortality (8). Troponin levels were not measured preoperatively in POSA, and studies have demonstrated chronically elevated troponin levels in severe OSA patients, independent of ischemic events diagnosed clinically or after review of electrocardiogram or echocardiogram (9). Perhaps anticipating this, the POSA authors performed a post-hoc analysis with "myocardial injury" replaced by "myocardial infarction" (clinical diagnosis as defined by an appropriate constellation of clinical and investigation results) and still found a significant association with severe OSA and the modified primary outcome (adjusted HR 2.04; 95\% CI, 1.33-2.99; $\mathrm{P}=0.001)$.

In addition to the preoperative sleep study, participants were assessed with STOP-Bang, an eight-item screening questionnaire for OSA that was originally validated for use in surgical populations (10), and later in multi-ethnic populations (11). Based on the STOP-Bang score, patients were stratified into low (score 0-2), moderate (score 3-4), or high (score 5-8) risk groups for OSA. While there was an association between increasing STOP-Bang risk group and severe OSA (C-index 0.714, 95\% CI, 0.670-0.758, $\mathrm{P}<0.001$ ), a high-risk STOP-Bang score had only modest utility for diagnosing severe OSA (sensitivity $58 \%$, specificity 78\%). Notwithstanding, participants with a highrisk STOP-Bang score, compared to those with a low-risk score, had an increased risk for the primary composite $\mathrm{CV}$ outcome (adjusted HR 1.68; 95\% CI, 1.11-2.54, $\mathrm{P}=0.01$ ). Thus, in locations where sleep monitoring is not available, there is a potential role for the STOP-Bang questionnaire in preoperative assessment, enabling more effective identification and triaging of high-risk patients.

The POSA study was generally well-designed and very carefully conducted. Major strengths are the prospective study design, recruitment of participants across multiple countries thereby enhancing the generalizability of the findings, objective measurement of sleep apnea using the same, validated sleep study device at all sites and a thorough assessment of primary endpoints by adjudicators who were blind to the sleep study results. However, there were also features that had the potential to weaken the study conclusions e.g., non-standardized post-surgical care between sites; and use of a Level III sleep study deviceApneaLink Plus ${ }^{\mathrm{TM}}$ —which does not measure sleep (12) and could lead to an underestimation of OSA severity. Also, while portable sleep monitoring data were collected and analyzed at the coordinating center, it is unknown what steps, if any, were taken to exclude participants with central sleep apnea (CSA). Rates of chronic opioid use are high in patients undergoing orthopedic surgery (13), which accounted for $30 \%$ of participants enrolled in POSA. CSA is commonly associated with chronic opioid use in a dosedependent fashion, and its impact on perioperative outcome is unknown. Patients on chronic opioids without CSA can develop CSA when acutely administered additional opioids (14). While the authors collected information on preoperative medication, information regarding preoperative opioid use was not provided.

The authors acknowledge the observational nature of their study findings; and that clinical trials will be needed in the future to determine if more intensive monitoring or therapy of OSA (e.g., continuous positive airway pressure, CPAP) can reduce postoperative CV risk. In this regard, and assuming that opioid-related central apnea was not a significant confounder, it is important to consider which pathophysiological mechanism or mechanisms associated with OSA might account for the increased postoperative $\mathrm{CV}$ risk that has been observed. OSA-associated hypoxia is perhaps the most obvious. As expected, participants with OSA were noted preoperatively to have a lower nadir peripheral capillary oxygen saturation $\left(\mathrm{SpO}_{2}\right)$, higher oxygen desaturation index (ODI, i.e., number of desaturation events $\geq 4 \%$ per hour of oximetry recording) and longer periods of time with $\mathrm{SpO}_{2}<80 \%$ or $<90 \%$. The degree of derangement in oxygen saturation was greatest in those with severe OSA. The application of oxygen postoperatively, predictably improved ODI in the groups with OSA, and postoperative ODI was not significantly associated with the primary outcome. However, ODI may not demonstrate the true extent of oxygen desaturation. While hypoxia was ameliorated by oxygen administration it was not abolished. Furthermore, participants who experienced a primary $\mathrm{CV}$ event had more severe oxygen desaturation (time spent with $\mathrm{SaO}_{2}<80 \%$ ) over the first 3 postoperative days (the time when most $\mathrm{CV}$ events were recorded) than those without $\mathrm{CV}$ complications, despite remaining on oxygen therapy for longer. Perhaps closer monitoring of postoperative arterial $\mathrm{SaO}_{2}$ and higher flow oxygen is one intervention that could be tested. However, caution is needed since it is possible that high flow oxygen could potentially be harmful to some patients with severe OSA. A 3-way interaction between severe OSA, postoperative opioids and oxygen administration may lead to respiratory depression, particularly in those with 
low ventilatory responsiveness and high arousal threshold $(15,16)$. Measurement of postoperative sleep variables or blood carbon dioxide $\left(\mathrm{CO}_{2}\right)$ were not included in the POSA study protocol. More work is required in this area. Certainly, postoperative monitoring for hypoventilation via continuous measurement of respiratory rate and end-tidal or transcutaneous $\mathrm{CO}_{2}$ should be considered for patients with known, untreated severe OSA.

A more logical OSA treatment is to maintain upper airway patency with CPAP or a similar device. By opening the upper airway, CPAP will alleviate hypoxia, lessen the chance of acute respiratory acidosis, reduce arousals and improve sleep architecture. It also abolishes the large negative intrathoracic swings that develop during breathing attempts against an obstructed airway and likely contribute to increased risk of atrial fibrillation, myocardial ischemia and heart failure by stretching the atria and increasing left ventricular mechanical afterload (17). Whether CPAP applied acutely postoperatively would be sufficient to prevent the excess postoperative $\mathrm{CV}$ risk attributed to OSA in POSA is unknown. OSA is a chronic proinflammatory disease that develops over many months to years and leads to excessive daytime sleepiness as well as night-time sympathetic hyperactivity and hypertension. These manifestations respond after several days to weeks of CPAP treatment but may not be fully reversed even after several months of treatment (18). The identification and treatment of severe OSA may need to occur several weeks prior to surgery to achieve the best results, not only for $\mathrm{CV}$ event prevention but for other adverse events (e.g., infections and unplanned reintubation and mechanical ventilation) demonstrated to be associated with OSA in POSA. These considerations will need to be taken into account in designing future clinical trials. And would undoubtedly present logistical challenges for surgeons and anesthetists responsible for the preoperative assessment and peri-operative management of patients undergoing major elective or emergency surgery.

Finally, a factor not addressed by the POSA authors is the contribution of supine positioning to their findings. As the supine position favors upper airway collapse, OSA is worsened (19). This may be another contributing factor to explain the increased ODI found postoperatively in patients with mild OSA in POSA. The majority of postoperative patients convalesce and sleep in the supine position due to various considerations (e.g., dressing access, surgical site location, etc.). A postoperative increase in the percentage of supine sleep has been previously observed in subjects undergoing elective surgical procedures (20). Furthermore, it has been demonstrated that the postoperative median AHI was is up to fourfold higher in supine sleep when compared to non-supine sleep (20).

To conclude, the POSA study is an important study which clarifies the postoperative risk of undiagnosed OSA patients. While not devoid of limitations, it furthers our understanding of the impact of OSA on patients in the surgical context, and strengthens existing anesthetic practice guidelines. While severe OSA has been shown by the POSA study to be associated with an increase in adverse postoperative $\mathrm{CV}$ outcomes, definitive proof of a causal relationship will require randomized controlled trials demonstrating that treatment of OSA can reduce postoperative CV risk. The POSA results suggest that the postoperative application of supplemental oxygen alone may not be sufficient to mitigate the OSA-associated risk. In the meantime, it would seem prudent to advise surgical patients who have been diagnosed with severe OSA and prescribed OSA treatment (e.g., CPAP) to use the therapy diligently in the run up to surgery, and ensure that they continue it postoperatively. In situations where a diagnosis of OSA has not been made but severe disease is suspected, screening of high CV risk surgical patients with the STOP-BANG questionnaire may be useful in identifying those who should have a preoperative sleep study or more in-depth postoperative monitoring. At present, the identification and therapy (as opposed to identification alone) of OSA is routine only in patients undergoing bariatric surgery. The next logical step is to determine if the treatment of newly diagnosed severe OSA in the perioperative period will mitigate the risk of adverse cardiovascular outcomes in high cardiovascular risk patients undergoing other types of major surgery.

\section{Acknowledgments}

R. Doug McEvoy is the recipient of a Practitioner Fellowship from the Australian National Health and Medical Research Council.

\section{Footnote}

Conflicts of Interest: The authors have no conflicts of interest to declare.

Ethical Statement: The authors are accountable for all aspects of the work in ensuring that questions related 
to the accuracy or integrity of any part of the work are appropriately investigated and resolved.

\section{References}

1. Sánchez-de-la-Torre M, Campos-Rodriguez F, Barbe F. Obstructive sleep apnoea and cardiovascular disease. Lancet Respir Med 2013;1:61-72.

2. Subramani $Y$, Singh $M$, Wong J, et al. Understanding Phenotypes of Obstructive Sleep Apnea: Applications in Anesthesia, Surgery, and Perioperative Medicine. Anesth Analg 2017;124:179-91.

3. Chung F, Memtsoudis SG, Ramachandran SK, et al. Society of Anesthesia and Sleep Medicine Guidelines on Preoperative Screening and Assessment of Adult Patients With Obstructive Sleep Apnea. Anesth Analg 2016;123:452-73.

4. Memtsoudis SG, Stundner O, Rasul R, et al. The impact of sleep apnea on postoperative utilization of resources and adverse outcomes. Anesth Analg 2014;118:407-18.

5. Mokhlesi B, Hovda MD, Vekhter B, et al. Sleep-disordered breathing and postoperative outcomes after elective surgery: analysis of the nationwide inpatient sample. Chest 2013;144:903-14.

6. Mutter TC, Chateau D, Moffatt M, et al. A matched cohort study of postoperative outcomes in obstructive sleep apnea: could preoperative diagnosis and treatment prevent complications? Anesthesiology 2014;121:707-18.

7. Chan MT, Wang CY, Seet E, et al. Association of Unrecognized Obstructive Sleep Apnea With Postoperative Cardiovascular Events in Patients Undergoing Major Noncardiac Surgery. JAMA 2019;321:1788-98.

8. Devereaux PJ, Biccard BM, Sigamani A, et al. Association of Postoperative High-Sensitivity Troponin Levels With Myocardial Injury and 30-Day Mortality Among Patients Undergoing Noncardiac Surgery. JAMA 2017;317:1642-51.

9. Lui MMS, Tse HF, Mak JCW, et al. Untreated Obstructive
Sleep Apnea Is Associated With Myocardial Injury Independent of Blood Pressure Control in Hypertension. J Clin Sleep Med 2018;14:1841-7.

10. Chung F, Subramanyam R, Liao P, et al. High STOPBang score indicates a high probability of obstructive sleep apnoea. Br J Anaesth 2012;108:768-75.

11. Nagappa M, Liao P, Wong J, et al. Validation of the STOP-Bang Questionnaire as a Screening Tool for Obstructive Sleep Apnea among Different Populations: A Systematic Review and Meta-Analysis. PLoS One 2015;10:e143697.

12. Auckley D, Memtsoudis S. Unrecognized Obstructive Sleep Apnea and Postoperative Cardiovascular Complications: A Wake-up Call. JAMA 2019;321:1774-6.

13. Hilliard PE, Waljee J, Moser S, et al. Prevalence of Preoperative Opioid Use and Characteristics Associated With Opioid Use Among Patients Presenting for Surgery. JAMA Surg 2018;153:929-37.

14. Mogri M, Khan MIA, Grant BJ, et al. Central sleep apnea induced by acute ingestion of opioids. Chest 2008;133:1484-8.

15. Lam KK, Kunder S, Wong J, et al. Obstructive sleep apnea, pain, and opioids: is the riddle solved? Curr Opin Anaesthesiol 2016;29:134-40.

16. Doufas AG. Obstructive sleep apnea, pain, and opioid analgesia in the postoperative patient. Current Anesthesiology Reports 2014;4:1-9.

17. Geovanini GR, Lorenzi-Filho G. Cardiac rhythm disorders in obstructive sleep apnea. J Thorac Dis 2018;10:S4221-30.

18. Torres G, Sanchez-de-la-Torre M, Barbe F. Relationship Between OSA and Hypertension. Chest 2015;148:824-32.

19. Joosten SA, O'Driscoll DM, Berger PJ, et al. Supine position related obstructive sleep apnea in adults: pathogenesis and treatment. Sleep Med Rev 2014;18:7-17.

20. Chung F, Liao P, Elsaid H, et al. Factors associated with postoperative exacerbation of sleep-disordered breathing. Anesthesiology 2014;120:299-311.
Cite this article as: Oh A, Mukherjee S, McEvoy RD. Unrecognized obstructive sleep apnea in surgery: we can't afford to sleep on it any longer. J Thorac Dis 2019;11(11):E235E238. doi: $10.21037 /$ jtd.2019.10.11 